\title{
ALEXANDER MODULES OF LINKS WITH ALL LINKING NUMBERS ZERO
}

\author{
M. L. PLATT
}

\begin{abstract}
In this paper we characterize the Alexander modules of links resulting from a surgical modification on the trivial link of any number of components. Using the presentation matrix obtained, we derive some properties of the Alexander polynomials of such links.
\end{abstract}

1. In [B, N, R, L, L-1 and L-2] the process of surgical modification of classical links is studied. [B and $\mathbf{N}$ ] consider the case of two-component links and give answers in terms of a change in the presentation matrix of the Alexander invariant $B$. The general case of the Alexander module $A$ (of which $B$ is a submodule) is studied in [L-1] but only after $A$ has been localized.

In the present paper, the effect of a surgical modification of the trivial link of any multiplicity is studied. The primary results are Theorems A and B. In Theorem A, a presentation matrix for such links is given. In Theorem B, it is shown that any matrix satisfying the requirements of Theorem A can be realized as the presentation matrix of a link all of whose linking numbers are zero.

Finally, the matrix given in Theorem A is examined in more detail. The Alexander polynomial of such links is determined from the matrix and is shown to be highly divisible by the augmentation ideal.

These results from part of the author's Ph.D. thesis which was written at Brandeis University under the supervision of Jerome P. Levine. The author wishes to thank Professor Levine for much help and encouragement.

2. A classical link of multiplicity $n$ is a smooth, ordered, oriented submanifold of $S^{3}$ consisting of $n$ disjoint circles, say $L=L_{1} \cup \cdots \cup L_{n}$. Let $N_{i}$ be a tubular neighborhood of $L_{i}$ chosen so that $N_{i} \cap N_{j}=\varnothing$ if $i \neq j$. Choose a basepoint $x_{i} \in \partial N_{i}$ and meridian and longitude circles in $\partial N_{i}$ which intersect at $x_{i}$ and are oriented so that the intersection number $m_{i} \cdot l_{i}=+1$ in $\partial N_{i}$. In addition, $l_{i}$ is to be oriented in the same direction as $L_{i}$ with linking number $\operatorname{lk}\left(l_{i}, L_{i}\right)=0$.

Let $x_{0}$ be a basepoint in the interior of $X=\overline{S^{3}-U N_{i}}$. For each $i$, choose an oriented arc $a_{i}$ from $x_{0}$ to $x_{i}$ in $X$. The elements $\alpha_{i}$ and $\beta_{i}$ in $\pi_{1} X$ represented by $a_{i} * m_{i} * a_{i}^{-1}$ and $a_{i} * l_{i} * a_{i}^{-1}$ are referred to as the meridians and longitudes of the link $L$. The pair $\left(\alpha_{i}, \beta_{i}\right)$ is well defined up to simultaneous conjugation by an element of $\pi_{1} X$.

By Alexander duality, $H_{1}(X) \approx \mathrm{Z}^{n}$ with a basis defined by the meridians of the link. This basis allows the identification of the group ring $\mathrm{Z}\left[H_{1}(X)\right]$ with $\Lambda_{n}=\mathbf{Z}\left[t_{1}, t_{1}^{-1}, \ldots, t_{n}, t_{n}^{-1}\right]$. There is a natural involution of $\Lambda_{n}$ (denoted with an

Received by the editors January 7, 1986.

1980 Mathematics Subject Classification (1985 Revision). Primary 57M25; Secondary 57M10. 


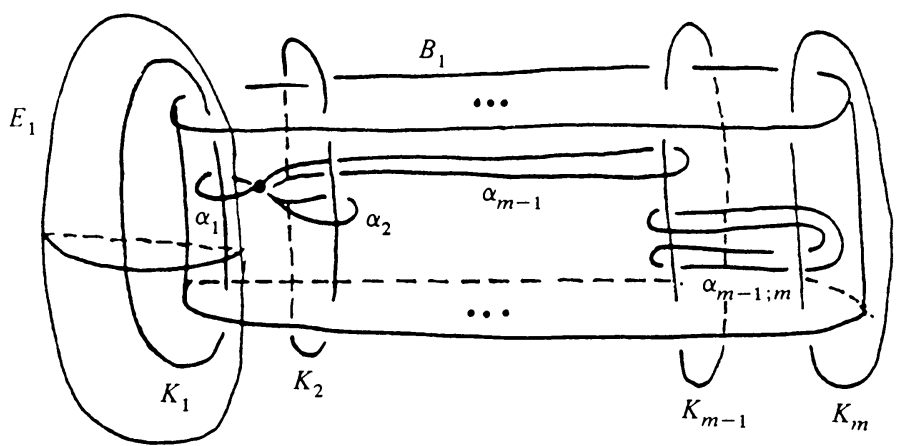

FIGURE

overbar) which maps $t_{i} \rightarrow t_{i}^{-1}$. The augmentation of $\Lambda_{n}$ is given by $\varepsilon: \Lambda_{n} \rightarrow \mathbf{Z}$ where $\varepsilon\left(t_{i}\right)=1$.

The homomorphism $h: \pi_{1} X \rightarrow H_{1}(X)$ defines a regular covering space, the universal abelian covering $p: \tilde{X} \rightarrow X$ with $\mathbf{Z}^{n}$ as the group of covering translations. $A=A(L)=H_{1}\left(\tilde{X}, \tilde{x}_{0}\right)$ is a module over $\Lambda_{n}$ referred to as the Alexander module of $L$. An important submodule of $A$ is $B=H_{1}(\tilde{X})$. These modules are related by the Crowell exact sequence $[\mathbf{C}, \mathbf{H}]$

$$
0 \rightarrow B \stackrel{\psi}{\rightarrow} A \stackrel{\phi}{\rightarrow} M \rightarrow 0
$$

where $\phi$ is the boundary homomorphism $\phi: A \rightarrow H_{0}(\tilde{X})$. Here, $H_{0}(\tilde{X})$ is identified with $\Lambda_{n}$ by a choice of basepoint $\bar{x}_{0} \in \tilde{x}_{0}$ and $M$ is the augmentation ideal of $\Lambda_{n}$ generated by $t_{1}-1, \ldots, t_{n}-1$.

We will use the technique of surgical modification as described in [L and $\mathbf{L}-\mathbf{1}]$. Let $S=\left\{\sigma_{1}, \ldots, \sigma_{r}\right\}$ be a collection of smooth disjoint circles imbedded in $S^{3}$ satisfying:

(i) $\sigma_{i} \cap L_{j}=\varnothing$ for all $i, j$,

(ii) $\operatorname{lk}\left(\sigma_{i}, L_{j}\right)=0$ for all $i, j$,

(iii) $\left\{\sigma_{i}\right\}$ considered as a link in $S^{3}$ is trivial.

Choose a normal framing which winds around each $\sigma_{i}$ once in the positive direction and do surgery on $S^{3}$ along $\left\{\sigma_{i}\right\}$. The resulting manifold $\Sigma$ is diffeomorphic to $S^{3}$ by (iii). The image of $L$ in $\Sigma$ is the surgical modification $L_{S}$.

From now on, we let $L_{0}$ denote the trivial link of $n$-components in $S^{3}$ with $X_{0}=\overline{S^{3}-\bigcup N_{i}}$ and universal abelian cover $\tilde{X}_{0}$. Let $\mu_{i}$ denote a lift of the $i$ th meridian of $L_{0}$ to $\tilde{X}_{0}$. We note that $\sum_{i=1}^{k} \lambda_{i} \mu_{i}$ is a cycle in $\tilde{X}_{0}$ if and only if $\sum_{i=1}^{k}\left(t_{i}-1\right) \lambda_{i}=0$. This is a direct consequence of the Crowell sequence (1). $A_{0}=$ $A\left(L_{0}\right)$ is a free $\Lambda_{n}$-module generated by $e_{1}, \ldots, e_{n}$ where $e_{i}$ is represented by $\mu_{i}$. $B_{0}=B\left(L_{0}\right)$ is generated by elements $e_{i j}(i<j)$ where $\psi\left(e_{i j}\right)=\left(t_{j}-1\right) e_{i}+\left(1-t_{i}\right) e_{j}$. There are relations among the $e_{i j}$. Namely, for $i<j<k,\left(t_{k}-1\right) e_{i j}+\left(1-t_{j}\right) e_{i k}+$ $\left(t_{i}-1\right) e_{j k}=0$. We also have $H_{2}\left(\tilde{X}_{0}, \tilde{x}_{0}\right) \approx H_{2}\left(\tilde{X}_{0}\right)$ is generated by $(n-1)$ twospheres. Let $E_{i}$ denote the two-sphere in $S^{3}$ which contains only the $i$ th component of $L_{0}$ and excludes the basepoint of $X_{0}$. The intersection pairings of $E_{i}$ and $e_{j}$ can be determined by considering $E_{i}$ as an element of $H_{2}\left(\tilde{X}_{0}-\tilde{x}_{0}\right) \approx H_{C}^{1}\left(\tilde{X}_{0}, \tilde{x}_{0}\right)$ and are given by $E_{i} \cdot e_{j}=\left(t_{i}-1\right) \delta_{i j}$. In $S^{3}$, these elements can be represented as shown 
in the Figure (see [L-2] for more details). Note that $\left\{e_{i j}\right\}_{i<j}$ may be represented so that when considered as a link, it is trivial. For $L_{S}$, we adopt the notation $X=\overline{\Sigma-\bigcup N_{i}}$ with universal abelian cover $\tilde{X}$.

With the conditions as above we have

THEOREM A. Let $L_{S}$ be a surgical modification of $L_{0}$, the trivial link of $n$ components. The Alexander module of $L, A=A(L)$ has a presentation with generators $e_{1}^{\prime \prime}, \ldots, e_{n}^{\prime \prime}, \alpha_{1}^{\prime \prime}, \ldots, \alpha_{r}^{\prime \prime}$ and relations

$$
\sum_{j=1}^{n}\left(t_{i}-1\right) \overline{\lambda_{j i}} \alpha_{j}^{\prime \prime}=0 \quad \text { for } 1 \leq i \leq n
$$

and

$$
\sum_{j=1}^{n} \lambda_{i j} e_{i}^{\prime \prime}+\sum_{j=1}^{k} \gamma_{i j} \alpha_{j}^{\prime \prime}=0 \quad \text { for } 1 \leq i \leq r
$$

where

(i) $\sum_{j=1}^{n}\left(t_{j}-1\right) \lambda_{i j}=0$,

(ii) $\gamma_{i j}=\overline{\gamma_{j i}}$,

(iii) $\varepsilon\left(\gamma_{i j}\right)=\delta_{i j}$.

PROOF. The proof is similar to that of Bailey's Theorem as given in [L-2].

Let $S=\left\{\sigma_{1}, \ldots, \sigma_{r}\right\}$ be the set of circles used to perform the surgical modification and let $\tilde{\sigma}_{i 1}$, be any lift of $\sigma_{i}$ to $\tilde{X}_{0}$. Let $Y$ be the complement of $S$ in $X_{0}$ and $\tilde{Y}$ the covering of $Y$ inherited from $\widetilde{X}_{0}$. In order to compute $H_{1}\left(\tilde{Y}, \tilde{x}_{0}\right)$ consider the exact sequence of the triple $\left(\tilde{X}_{0}, \tilde{Y}, \tilde{x}_{0}\right)$.

$$
\rightarrow H_{2}\left(\tilde{X}_{0}, \tilde{x}_{0}\right) \rightarrow H_{2}\left(\tilde{X}_{0}, \tilde{Y}\right) \rightarrow H_{1}\left(\tilde{Y}, \tilde{x}_{0}\right) \rightarrow A_{0} \rightarrow 0 .
$$

Since $A_{0}$ is free and $H_{2}\left(\tilde{X}_{0}, \tilde{Y}\right)$ is free on $\left\{\alpha_{i}\right\}$,

$$
H_{1}\left(\tilde{Y}, \tilde{x}_{0}\right) \approx A_{0} \oplus H_{2}\left(\tilde{X}_{0}, \tilde{Y}\right) / \operatorname{Im} H_{2}\left(\tilde{X}_{0}, \tilde{x}_{0}\right) .
$$

Since $\sigma_{i}$ lifts to an imbedded circle $\tilde{\sigma}_{i 1}$ in $\tilde{X}_{0},(1)$ implies

$$
\tilde{\sigma}_{i 1}=\sum_{j=1}^{n} \lambda_{i j} e_{j} \quad \text { with } \sum_{j=1}^{n}\left(t_{j}-1\right) \lambda_{i j}=0 .
$$

From this, it is clear that $H_{1}\left(\tilde{Y}, \tilde{x}_{0}\right)$ is generated by $e_{1}^{\prime}, \ldots, e_{n}^{\prime}$ where $e_{i}^{\prime} \rightarrow e_{i}$ under the inclusion $Y \rightarrow X_{0}$ and by $\alpha_{1}, \ldots, \alpha_{r}$ where $\alpha_{i}$ is represented by a small circle which links $\tilde{\sigma}_{i 1}$ simply.

The relations in $H_{1}\left(\tilde{Y}, \tilde{x}_{0}\right)$ are given by the images of elements in $H_{2}\left(\tilde{X}_{0}, \tilde{x}_{0}\right)$. Since $H_{2}\left(\widetilde{X}_{0}, \tilde{x}_{0}\right)$ is freely generated by $(n-1)$ of the $E_{i}$, there is one relation for each $E_{i}$ given by the intersection pairing. By using all of the $E_{i}$, we obtain $n$ relations, $R_{1}, \ldots, R_{n}$, one of which is redundant. That is,

$$
R_{i}: \sum_{j=1}^{r} \gamma_{i j} \alpha_{j}=0 \quad \text { where } \gamma_{i j}=E_{i} \cdot \tilde{\sigma}_{j 1} .
$$

Using (3) we obtain

$$
\gamma_{i j}=E_{i} \cdot\left(\sum_{k=1}^{n} \lambda_{j k} e_{k}\right)=\sum_{k=1}^{n} \overline{\lambda_{j k}}\left(E_{i} \cdot e_{k}\right)=\left(t_{i}-1\right) \overline{\lambda_{j i}}
$$


Thus, the relations are

$$
\sum_{j=1}^{r}\left(t_{i}-1\right) \overline{\gamma_{j i}} \alpha_{j}=0, \quad 1 \leq i \leq n,
$$

and $H_{1}\left(\tilde{Y}, \tilde{x}_{0}\right)$ has a presentation with generators $e_{1}^{\prime}, \ldots, e_{n}^{\prime}, \alpha_{1}, \ldots, \alpha_{k}$ and relations (5). (Note that the rows of the corresponding presentation matrix are linearly dependent since $\sum_{i=1}^{n} E_{i}=0$ in $H_{2}\left(\tilde{X}_{0}, \tilde{x}_{0}\right)$.)

To compute $A$, we examine the exact sequence of the triple $\left(\tilde{X}, \tilde{Y}, \tilde{x}_{0}\right)$

$$
\rightarrow H_{2}(\tilde{X}, \tilde{Y}) \stackrel{\partial^{*}}{\longrightarrow} H_{1}\left(\tilde{Y}, \tilde{x}_{0}\right) \stackrel{i_{*}}{\longrightarrow} A \rightarrow 0 .
$$

From this, we see that $A \approx H_{1}\left(\tilde{Y}, \tilde{x}_{0}\right) / \partial_{*} H_{2}(\tilde{X}, \tilde{Y})$ is generated by $e_{1}^{\prime \prime}, \ldots, e_{n}^{\prime \prime}, \alpha_{1}^{\prime}$, $\ldots, \alpha_{r}^{\prime}$ the images of $e_{i}^{\prime}, \ldots, e_{n}^{\prime}, \alpha_{1}, \ldots, \alpha_{r}$ under the inclusion $Y \rightarrow X$. In addition, there are new relations obtained by attaching a disc along $\tilde{\sigma}_{i 1}^{\prime}$, where the circle $\tilde{\sigma}_{i 1}^{\prime}$ is the result of translating $\tilde{\sigma}_{i 1}$ along the vector field used in the surgery. $H_{2}(\tilde{X}, \tilde{Y})$ is generated by $d_{1}, \ldots, d_{r}$ where $d_{i}$ can be represented by a disc whose boundary is $\tilde{\sigma}_{i 1}^{\prime}$. The additional relations are given by

$$
R_{i}: i_{*} \partial_{*}\left(d_{i}\right)=0
$$

That is,

$$
\sum_{j=1}^{n} \lambda_{i j} e_{j}^{\prime \prime}+\sum_{j=1}^{r} \gamma_{i j}^{\prime} \alpha_{j}^{\prime}=0 \quad \text { for some } \gamma_{i j}^{\prime} .
$$

That the coefficient of $e_{j}^{\prime \prime}$ is $\lambda_{i j}$ follows from (3). From (1) and (3) we may write

$$
\tilde{\sigma}_{i}^{\prime}=\sum_{s<t} \gamma_{i s t} e_{s t}+\sum_{j=1}^{r} \gamma_{i j}^{\prime} \alpha_{j}^{\prime \prime}
$$

We need to show that the correct original choice of $\left\{e_{s t}\right\}_{s<t}$ and corresponding $\left\{e_{j}\right\}$ results in the following properties:

$$
\text { (i) } \gamma_{i j}^{\prime}=\overline{\gamma_{j i}^{\prime}}, \quad \text { (ii) } \varepsilon\left(\gamma_{i j}^{\prime}\right)=\delta_{i j} \text {. }
$$

We need to use the following lemma from [L-2].

LEMMA. Suppose $M$ is a compact oriented 3-manifold, $\widetilde{M} \rightarrow M$ a regular covering with $\pi$ as the group of covering transformations. Let $T_{1}, \ldots, T_{n}$ be tori components of $\partial M$ which lift to $\widetilde{T}_{i} \subseteq \widetilde{M}$ trivially covering $T_{i}$, for each $i$. Let $\alpha_{i}, \beta_{i}$ be the canonical generators of $H_{1}\left(\widetilde{T}_{i}\right)$ represented by meridian and longitude circles satisfying $\alpha_{i} \cdot \alpha_{j}=0=\beta_{i} \cdot \beta_{j}$ and $\alpha_{i} \cdot \beta_{j}=\delta_{i j}$. If

$$
\sum_{j} \lambda_{i j} i_{*}\left(\alpha_{j}\right)+\sum_{j} \mu_{i j} i_{*}\left(\beta_{j}\right)=0, \quad i=1, \ldots, n,
$$

is any set of relations in $H_{1}(\widetilde{M}), i: \widetilde{T}_{i} \subseteq \widetilde{M}$, then, for any $i, j$

$$
\sum_{s} \lambda_{i s} \overline{\mu_{j s}}=\sum_{s} \mu_{i s} \overline{\lambda_{j s}} \quad \text { where } \mu \rightarrow \bar{\mu}
$$

is the usual conjugation in $\mathbf{Z} \pi$. 
To apply the lemma, we remove tubular neighborhoods of the loops $\tau_{i j}$, representing $e_{i j}$, from $Y$ to obtain a new manifold $W$. The new equations are

$$
\tilde{\sigma}_{i 0}^{\prime}=\sum_{s<t} \gamma_{i s t} e_{s t 0}+\sum_{J} \gamma_{i j}^{\prime} \alpha_{j 0}+\sum_{s<t} \overline{\mu_{i s t}} C_{s t} \quad \text { in } H_{1}(\widetilde{W})
$$

where

(i) $C_{s t}$ is represented by a meridian of the newly removed neighborhood of $\tau_{s t}$,

(ii) $e_{s t o}$ is represented by $\tilde{\tau}_{s t}^{\prime}$, a translate of $\tilde{\tau}_{s t}$ into $\widetilde{W}$ chosen so that $\mathrm{lk}\left(\tau_{s t}^{\prime}, \tau_{s t}\right)=$ 0 in $S^{3}$,

(iii) $\alpha_{j 0} \rightarrow \alpha_{j}^{\prime \prime}$ and $\tilde{\sigma}_{i 0}^{\prime} \rightarrow \tilde{\sigma}_{i}^{\prime}$.

Applying the lemma to (10) gives

$$
\gamma_{i j}^{\prime}+\sum_{s<t} \gamma_{i s t} \mu_{j s t}=\overline{\gamma_{j i}^{\prime}}+\sum_{s<t} \overline{\mu_{i s t}} \overline{\gamma_{j s t}}
$$

assuming that $\left\{\alpha_{j}^{\prime \prime}\right\}$ and $\left\{c_{s t}\right\}$ are oriented correctly. Now we replace the original choice of $e_{s t}$ with $\varepsilon_{s t}=e_{s t}-\sum_{j=1}^{r} \mu_{j s t} \alpha_{j}^{\prime \prime}$. We note that

$$
\begin{aligned}
\tilde{\sigma}_{i}^{\prime} & =\sum_{s<t} \gamma_{i s t}\left(\varepsilon_{s t}+\sum_{j=1}^{r} \mu_{j s t} \alpha_{j}^{\prime \prime}\right)+\sum_{j=1}^{r} \gamma_{i j}^{\prime} \alpha_{j}^{\prime \prime} \\
& =\sum_{s<t} \gamma_{i s t} \varepsilon_{s t}+\sum_{j=1}^{r}\left(\gamma_{i j}^{\prime}+\sum_{s<t} \gamma_{i s t} \mu_{j s t}\right) \alpha_{j}^{\prime \prime} .
\end{aligned}
$$

Hence $\gamma_{i j}^{\prime}$ is replaced by $\gamma_{i j}=\gamma_{i j}^{\prime}+\sum_{s<t} \gamma_{i s t} \mu_{j s t}$ so property (9)(i) is satisfied.

To verify (9)(ii) we project everything to $W \subseteq S^{3}$ and note

(a)

$$
\begin{aligned}
\varepsilon\left(\mu_{i s t}\right) & =\operatorname{lk}\left(\tau_{s t}, \sigma_{i}-\sum_{u<v} \varepsilon\left(\gamma_{i u v}\right) \tau_{u v}^{\prime}\right) \\
& =\operatorname{lk}\left(\tau_{s t}, \sigma_{i}\right)-\sum_{u<v} \varepsilon\left(\gamma_{i u v}\right) \operatorname{lk}\left(\tau_{s t}, \tau_{u v}^{\prime}\right) \\
& =\operatorname{lk}\left(\tau_{s t}, \sigma_{i}\right) \quad\left(\text { recalling that the link formed by }\left\{\tau_{s t}\right\} \text { is trivial }\right),
\end{aligned}
$$

(b)

$$
\begin{aligned}
\varepsilon\left(\gamma_{i j}^{\prime}\right) & =\operatorname{lk}\left(\sigma_{j}, \sigma_{i}^{\prime}-\sum_{u<v} \varepsilon\left(\gamma_{i u v}\right) \tau_{u v}^{\prime}\right) \\
& =\operatorname{lk}\left(\sigma_{j}, \sigma_{i}^{\prime}\right)-\sum_{u<v} \varepsilon\left(\gamma_{i u v}\right) \operatorname{lk}\left(\sigma_{j}, \tau_{u v}^{\prime}\right) \\
& =\delta_{i j}-\sum_{u<v} \varepsilon\left(\gamma_{i u v}\right) \varepsilon\left(\mu_{j u v}\right) .
\end{aligned}
$$

Together (a) and (b) imply

$$
\varepsilon\left(\gamma_{i j}\right)=\varepsilon\left(\gamma_{i j}^{\prime}\right)-\sum_{u<v} \gamma_{i u v} \mu_{j u v}=\delta_{i j}
$$

and property (9)(ii) is satisfied concluding the proof of Theorem A.

We now prove the following theorem. 
THEOREM B. Let $\left(\lambda_{i j}\right)$ be an $(m \times m)$-matrix over $\Lambda_{n}$ satisfying

(i) $\lambda_{i j}=0$ for $1 \leq i, j \leq n$,

(ii) $\sum_{j=1}^{r}\left(t_{i}-1\right) \lambda_{i j}=0$ for $i>n$,

(iii) $\lambda_{i j}=\left(t_{i}-1\right) \overline{\lambda_{j i}}$ for $i>n$ and $1 \leq j \leq n$,

(iv) $\lambda_{i j}=\overline{\lambda_{j i}}$ for $i, j>n$,

(v) $\varepsilon\left(\lambda_{i j}\right)=\delta_{i j}$ for $i, j>n$.

Then there exists a link with all linking numbers zero, whose module $A$ has a presentation $\left\{\alpha_{1}, \ldots, \alpha_{m}: \sum_{j=1}^{m} \lambda_{i j} \alpha_{j}=0\right\}$.

PROOF. The proof is similar to that of Theorem A, and we use the same notation. Let $X_{0}$ be the complement of the $n$-component trivial link with generators $e_{i}$ of $H_{1}\left(\tilde{X}_{0}, \tilde{x}_{0}\right)$. Let $\left\{\sigma_{i}\right\}_{i=n+1}^{m}$ be disjoint imbedded circles in $X_{0}$ which lift to $\left\{\tilde{\sigma}_{i}\right\}$ in $\widetilde{X}_{0}$ such that $\tilde{\sigma}_{i}$ represents $\sum_{j=1}^{n} \lambda_{i j} e_{j}$. Properties (ii) and (iii) insure that $\sigma_{i}$ is an imbedded circle in $\tilde{X}_{0}$. Furthermore, situate $\left\{\sigma_{i}\right\}$ so that $\left\{\sigma_{i}\right\}$ is a trivial link in $S^{3}$. Give each $\sigma_{i}$ the normal framing which winds around once and do surgery. $H_{1}\left(\tilde{Y}, \tilde{x}_{0}\right)$ and $H_{1}\left(\tilde{X}, \tilde{x}_{0}\right)$ are computed precisely as before. We obtain relations

$$
\sum_{j=1}^{n} \lambda_{i j} e_{j}^{\prime \prime}+\sum_{j=n+1}^{m} \lambda_{i j}^{\prime} \alpha_{j}^{\prime \prime}=0
$$

for some $\gamma_{i j}^{\prime} \in \Lambda_{n}$. The $\gamma_{i j}^{\prime}$ may not be the $\lambda_{i j}$ specified in the hypothesis. However, by the proper choice of $e_{i j}$ and $e_{i}$ we have $\gamma_{i j}^{\prime}=\overline{\gamma_{j i}^{\prime}}$ and $\varepsilon\left(\gamma_{i j}^{\prime}\right)=\delta_{i j}$. Finally, the $\gamma_{i j}^{\prime}$ can be altered in order to realize the specified $\lambda_{i j}$ by using a combination of the elementary changes described in [L-2].

3. The Alexander polynomial $\Delta$ of the link can be determined from a presentation matrix for $A$. It is the greatest common divisor of all the $(n+m-1)$-minors. [T] has shown that $\Delta \in M^{2 n-3}$ in general when all linking numbers are zero. In Theorem $\mathrm{H}$, we show that in our situation $\Delta \in M^{3 n-4}$. Thus, the Borromean rings are not a surgical modification of the trivial 3-component link since the Alexander polynomial of the Borromean rings is $\left(t_{1}-1\right)\left(t_{2}-1\right)\left(t_{3}-1\right)$. We adopt the following notation.

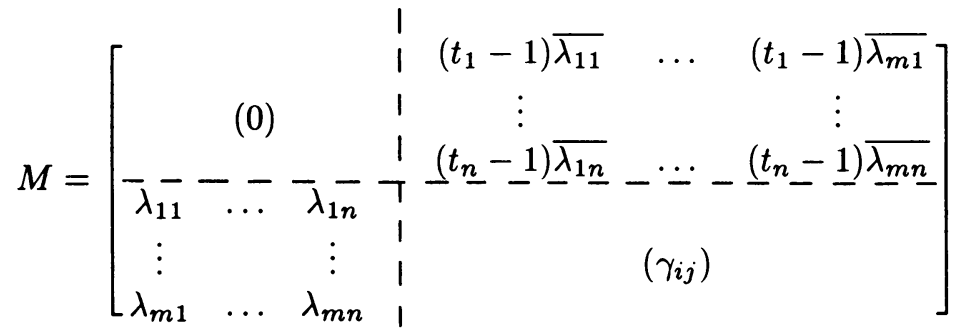

where $\sum_{j}\left(t_{j}-1\right) \lambda_{i j}=0$. Let $M_{i j}$ denote the $(n+m-1)$-submatrix obtained by deleting the $i$ th row and $j$ th column of $M$. Then,

Proposition C. (i) $\left|M_{i j}\right|=0$ if $i>n$ or $j>n$,

(ii) $\left(t_{j}-1\right)\left|M_{i i}\right|= \pm\left(t_{i}-1\right)\left|M_{i j}\right|$,

(iii) $\left|M_{i j}\right|=\left(\right.$ unit) $\left|M_{j j}\right|$ for $i, j \leq n, i \neq j$.

Thus, $M_{i i}$ and $M_{j j}$ are related. 
ProOF. (i) If $i>n$ (resp. $j>n$ ), then $M_{i j}$ contains the first $n$ rows (resp. columns) of $M$ which are linearly dependent since $\sum_{j}\left(t_{j}-1\right) \lambda_{i j}=0$.

(ii) If $i, j \leq n$, column 1 can be replaced by a linear combination of the first $n$ columns of $M$ excluding column 1 . More precisely, denote by - that a row (column) has been deleted. Since $\sum_{j}\left(t_{j}-1\right) \lambda_{i j}=0$ for each $i$, we may write (using the relation $\sum_{r}\left(t_{r}-1\right) \lambda_{k r}=0$ in the last step)

$$
\begin{aligned}
& \left|M_{i i}\right|=\prod_{k \neq i}\left(t_{k}-1\right)\left|\begin{array}{cccccc} 
& & & \overline{\lambda_{11}} & \ldots & \overline{\lambda_{m 1}} \\
& & \vdots & & \vdots \\
\lambda_{11} & \ldots & \lambda_{1 n} & & \\
\vdots & & \vdots & & \left(\gamma_{i j}\right) \\
\lambda_{m 1} & \ldots & \lambda_{m n} & &
\end{array}\right|
\end{aligned}
$$

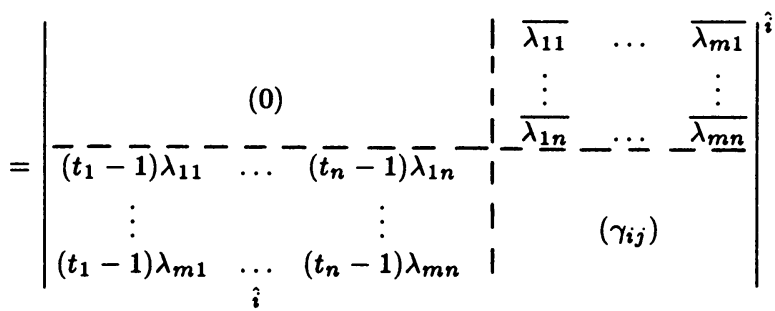

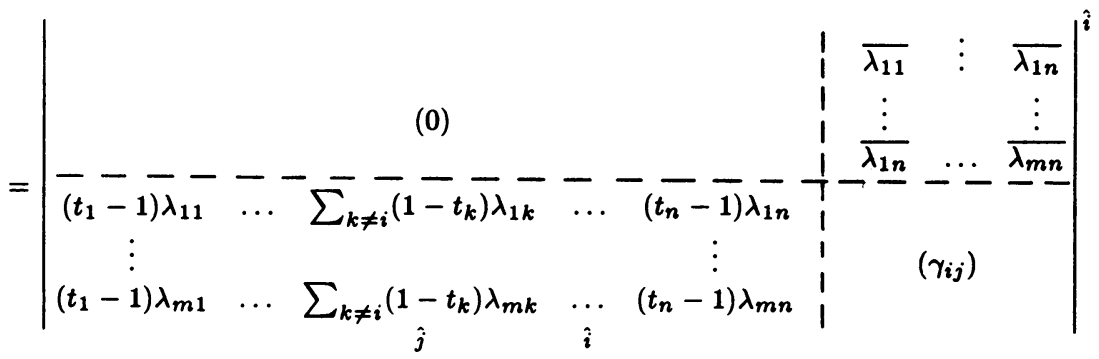

Now add to column $j$, the sum of the other $(n-2)$ initial columns.

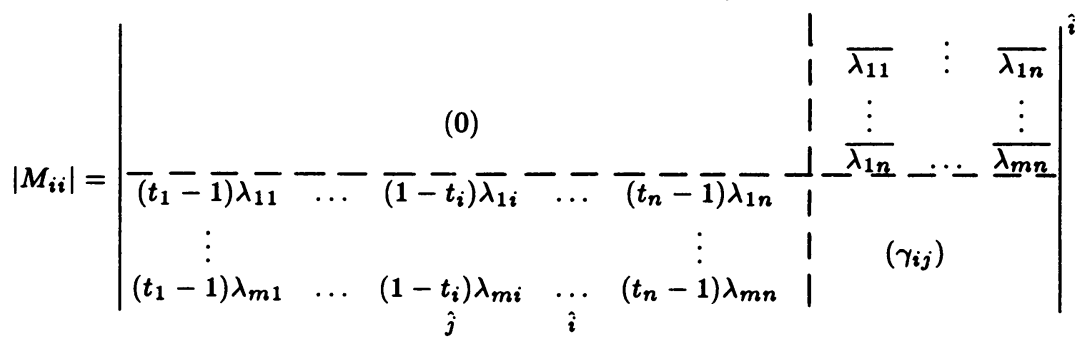

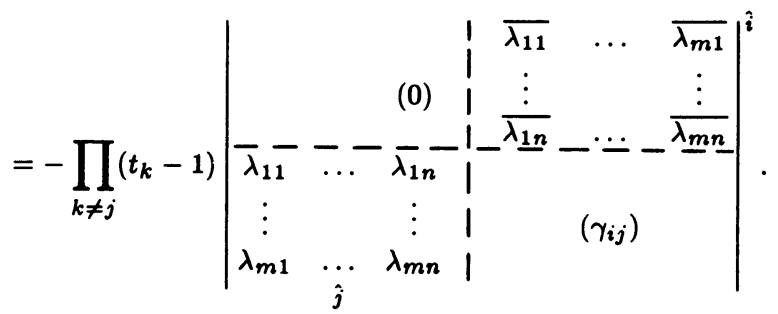


Thus,

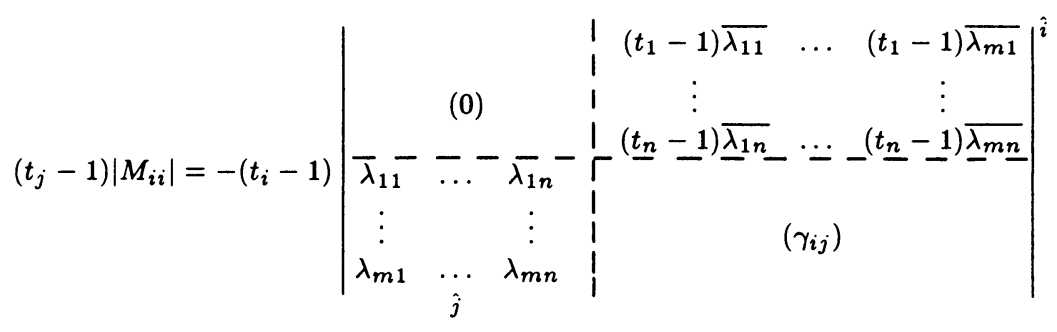

That is, $\left(t_{j}-1\right)\left|M_{i i}\right|= \pm\left(t_{i}-1\right)\left|M_{i j}\right|$, the sign depending on the parity of the permutation of the first $(n-1)$ columns.

(iii) This follows from a calculation similar to that of (ii) using the first $(n-1)$ rows of $M_{i j}$ and the conjugate relation $\sum_{j}\left(t_{j}^{-1}-1\right) \overline{\lambda_{i j}}=0$. The details are left to the reader.

COROllary D. If $m<(n-2),\left|M_{i i}\right|=0$ for all $i$. Consequently, the Alexander polynomial of the corresponding link is zero.

Proof. By Theorem $\mathrm{C}$, it is only necessary to consider $M_{i i}$ for $i \leq n$. This matrix has the form

$$
\left(\begin{array}{cc}
0_{(n-1) \times(n-1)} & *_{(n-1) \times m} \\
*_{m \times(n-1)} & *_{m \times m}
\end{array}\right) .
$$

The determinant is the sum of terms obtained by choosing an entry from each column and row. From the first $(n-1)$ columns, we must choose $(n-1)$ entries, each from a different row. However, there are at most $(n-2)$ nonzero choices, so at least one zero must be chosen. Thus, each term is zero, hence so is the sum.

An easy calculation gives the following corollaries which are also proven more generally in [C-1].

COROLlaRY E. If $i<n$, then $\left|M_{i i}\right|=\left(t_{i}-1\right)\left[\prod_{k}\left(t_{k}-1\right)\right] \Delta$ (up to a unit) for some $\delta \in \Lambda_{n}$.

COROllary F. The Alexander polynomial of the link $L_{S}$ is $A\left(t_{1}, \ldots, t_{n}\right)=$ $\left[\prod_{k}\left(t_{k}-1\right)\right] \Delta$.

We need

LEMMA G. If $\sum_{j}\left(t_{j}-1\right) \lambda_{j}=0$, then $\lambda_{j}$ is in the ideal generated by the $\left(t_{k}-1\right)$ for $k \neq j$. That is, $\lambda_{j} \in\left(\left(t_{1}-1\right), \ldots,\left(\widehat{t_{j}-1}\right), \ldots,\left(t_{n}-1\right)\right)$.

Proof. From the Koszul complex

$$
\begin{gathered}
\rightarrow \cdots\left(\begin{array}{l}
n \\
2
\end{array}\right) \Lambda_{n} \rightarrow n \Lambda_{n} \rightarrow \Lambda_{n} \rightarrow \mathbf{Z} \rightarrow 0 \\
\sum_{j}\left(t_{j}-1\right) \lambda_{j}=0 \quad \text { implies } \quad \lambda_{j}=\sum_{i} a_{i j}\left(t_{i}-1\right)
\end{gathered}
$$

with $a_{i j}=-a_{j i}$. Hence $a_{i i}=0$ and the result follows.

Using Lemma $\mathrm{G}$ we prove the following theorem. 
THEOREM H. The Alexander polynomial $A$ of any link obtained by a surgical modification of the trivial link is in $M^{3 n-4}$ where $M$ is the augmentation ideal.

Proof. It is enough to consider

$$
\left|M_{11}\right|=\left[\prod_{k=2}^{n}\left(t_{k}-1\right)\right] \Delta_{1}=\left(t_{1}-1\right)\left[\prod_{k=1}^{n}\left(t_{k}-1\right)\right] \Delta
$$

where

$$
\Delta_{1}=\left|\begin{array}{cc}
0 & \overline{\left(\lambda_{j i}\right)} \\
\left(\lambda_{i j}\right) & *
\end{array}\right| \quad(j \neq 1) .
$$

Each nonzero summand of $\Delta_{1}$ has the form

$$
\left(\lambda_{i_{2} 2} \ldots \lambda_{i_{m} m}\right)\left(\overline{\lambda_{2 k_{2}}} \ldots \overline{\lambda_{m k_{m}}}\right) \quad \text { (product of entries in } * \text { ) }
$$

where $i_{r} \neq i_{s}$ and $k_{r} \neq k_{s}$. Since each $\lambda_{i j} \in M$, the summands are in $M^{n-1}$. $M^{n-1}=M^{2 n-2}$. Since $\Delta_{1}=\left(t_{1}-1\right)^{2} \Delta, \Delta \in M^{2 n-4}$. Thus

$$
A=\left[\prod\left(t_{k}-1\right)\right] \Delta \in M^{n} \cdot M^{2 n-4}=M^{3 n-4} .
$$

We conclude with a result related to one of Kidwell [K]. Suppose $n=3$ and $A$ is the Alexander polynomial of any 3-component link for which all linking numbers are zero. Then $A$ can be factored as $A=\left(t_{1}-1\right)\left(t_{2}-1\right)\left(t_{3}-1\right) \Delta$. In $[\mathbf{K}]$ it is shown that $|\varepsilon(\Delta)|$ is a perfect square and that any square can occur.

For any surgical modification of the trivial link, we have

THEOREM I. $|\varepsilon(\Delta)|=0(n \geq 3)$.

Proof. $A \in M^{3 n-4}$ and $\Delta \in M^{2 n-4}$ and $2 n-4>0$.

\section{REFERENCES}

[B] J. H. Bailey, Alexander invariants of links, $\mathrm{Ph}$. D. thesis, Univ. of British Columbia, 1977.

[C] R. H. Crowell, Corresponding group and module sequences, Nagoya Math. J. 19 (1961), 27-40. [C-1] _ , Torsion in link modules, J. Math. Mech. 14 (1965), 289-298.

[H] J. A. Hillman, Alexander ideals of links, Lecture Notes in Math., vol. 895, Springer-Verlag, New York, 1981.

[K] M. Kidwell, On the Alexander polynomial of certain three component links, Proc. Amer. Math. Soc. 71 (1978), 351-353.

[L] J. P. Levine, A method for generating link polynomials, Amer. J. Math. 89 (1967), 69-84.

[L-1] _ The presentation matrix of a link module, preprint.

[L-2] _ $\ldots$, The module of a two-component link, Comment. Math. Helv. 57 (1982), 377-399.

[N] Y. Nakaniski, A surgical view of Alexander invariants of links, Math. Sem. Notes Kobe Univ. 8 (1980), 199-218.

[R] D. Rolfsen, Knots and links, Publish or Perish, Berkeley, Calif., 1976.

[T] L. Tradi, Linking numbers and elementary ideals of links, Trans. Amer. Math. Soc. 275 (1983). 01970

Department of Mathematics, Salem State College, Salem, Massachusetts 